\title{
A12
}

doi: 10.14232/ syrpharmacognosy.2021.a12

\section{Reports of illegal food supplements for weight loss in the European Rapid Alert System for Food and Feed (RASFF)}

\author{
Dorottya Koncz
}

Email: Koncz.Dorottya@stud.u-szeged.hu

Obesity is considered as an epidemic; thus the treatment is of major importance. Many customers are using dietary supplements to induce weight loss; however, the efficacy and safety of these products are often doubtful [1]. In the European Union, the Rapid Alert System for Food and Feed (RASFF) was put in place to provide food and feed control authorities with an effective tool to information exchange on measures taken responding to serious risks detected in relation to food or feed [2]. The reports of RASFF confirmed that adulterated products are on the market. These products often contain sympathomimetic drugs or herbal substances to enhance efficacy; therefore, the use of these supplements might pose a serious health risk for consumers. The aim of our work was to summarize the trends concerning adulterated food supplements associated with a warning released by the RASFF between 1988 and 2019, focusing on products with intended use as slimming agent. Data were extracted from the RASFF portal. In total, 299 $(11.7 \%)$ records of food supplements with quality problems were identified in the RASFF, and several of these products were marketed to facilitate weight loss. $182(60.8 \%)$ of these contained synthetic drug ingredients. Significant number of weight loss supplements is adulterated to satisfy patients' expectations. Hence, these products may cause serious adverse effects depending on the adulterants in it. The data presented here can be used as a basis for a suitable prediction model for the quality control of slimming products.

Supervisors: Dezső Csupor, Barbara Tóth

\section{Acknowledgements:}

The author is grateful for Orsolya Roza for her kind help during the preparation of the manuscript. The financial support of University of Szeged Open Access Fund is acknowledged.

\section{References}

[1] Ríos-Hoyo A and Gutiérrez-Salmeán G (2016) Current Obesity Reports 2016; 5:262-270.

[2] RASFF-Food and Feed Safety Alerts https:// ec.europa.eu/food/ safety/rasff/how_does_rasff_work/legal_basis_ en. [Accessed: 2020 Feb 15]. 